\title{
Protective effect of Dachengqi decoction on the pancreatic microcirculatory system in severe acute pancreatitis by down-regulating HMGB-TLR-4-IL-23-IL-17A mediated neutrophil activation by targeting SIRT1
}

\author{
Jia Wang ${ }^{1 \#}$, Yang Zou ${ }^{2 \#}$, Dan Chang ${ }^{2}$, Da-Qing Hong' ${ }^{2}$, Jiong Zhang ${ }^{2}$ \\ ${ }^{1}$ General Practice Center, Sichuan Provincial People's Hospital \& Sichuan Academy of Sciences, University of Electronic Science and Technology, \\ Chengdu, China; ${ }^{2}$ Division of Nephrology, Sichuan Provincial People's Hospital \& Sichuan Academy of Sciences, University of Electronic Science \\ and Technology, Chengdu, China \\ Contributions: (I) Conception and design: J Zhang; (II) Administrative support: DQ Hong; (III) Provision of study materials or patients: D Chang; (IV) \\ Collection and assembly of data: Y Zou; (V) Data analysis and interpretation: J Wang; (VI) Manuscript writing: All authors; (VII) Final approval of \\ manuscript: All authors. \\ \#These authors contributed equally to this work. \\ Correspondence to: Jiong Zhang; Da-Qing Hong. Division of Nephrology, Sichuan Provincial People's Hospital \& Sichuan Academy of Sciences, \\ University of Electronic Science and Technology, Chengdu, China. Email: zhangjiong831224@163.com; hongdaqing11@126.com.
}

Background: Dachengqi decoction (DCQD), one of classic prescription of Chinese herbal medicine has been widely used in clinic to treat severe acute pancreatitis (SAP). The damage of pancreatic microcirculation plays key pathogenesis of SAP. However, little is known about the molecular pharmacological activity of DCQD on pancreatic microcirculation in SAP.

Methods: Sodium taurodeoxycholate and cerulein were used to establish model of SAP in vitro and in vivo, respectively. The pancreatic pathological morphology, wet weight ratio, myeloperoxidase (MPO) activity, cell viability and microcirculatory function of the pancreas, as well as serum lipase and amylase expressions were evaluated. The expression levels of SIRT1, acety-HMGB1, TLR-4, HMGB1, IL-23, IL-17A, neutrophil chemokines (KC, LIX, and MIP-2), and inflammation-related factors (IL-6, IL-1 $\beta$, and TNF- $\alpha$ ), the translocation of HMGB1 and the interaction of SIRT-HMGB1 in the pancreas and serum were determined by ELISA real-time PCR, western blotting and immunoprecipitation.

Results: In vivo studies showed that DCQD or neutralizing antibody (anti-23p19 or anti-IL-17A) could all significantly decrease lipase, amylase activity, down-regulate the expression of CD68, Myeloperoxidase (MPO), wet/weight, IL-1 $\beta$, IL-6, TNF- $\alpha$, and neutrophil chemokines (KC, LIX, MIP-2), alleviate pathological injury and improve pancreatic microcirculatory function in rats with SAP. Furthermore, DCQD remarkably increased SIRT1 expression, promoted SIRT1 and HMGB1 combination, reduced HMGB1 translocation from nuclear to cytoplasm, and alleviated the expression of acetyl-HMGB1, HMGB1, IL-17A, TLR-4, and IL-23 in vitro and in vivo with SAP. However, the intervention with EX527 (SIRT1 inhibitor) or r-HMGB1 (recombinant HMGB1) obliviously reverses the above mentioned influence mentioned above of DCQD in SAP. In vitro, we confirmed that DCQD could decrease HMGB1 acetylation, migration, and release, and improve the decline of cell viability, SIRT1 expression and SIRI-HMGB1 combination induced by cerulean with promoting macrophage to release IL-23 by relying on the HMGB1/TLR-4 way.

Conclusions: DCQD treatment improves SAP-induced pancreatic microcirculatory dysfunction by inhibiting neutrophil-mediated inflammation via inactivating HMGB1-TLR-4-IL-23-IL-17A signaling by targeting SIRT1. 
Keywords: Severe acute pancreas (SAP); Dachengqi decoction (DCQD); neutrophil; HMGB1-TLR-4-IL-23-IL17A; pancreatic microcirculatory

Submitted Sep 05, 2021. Accepted for publication Oct 14, 2021.

doi: $10.21037 / \mathrm{gs}-21-655$

View this article at: https://dx.doi.org/10.21037/gs-21-655

\section{Introduction}

Severe acute pancreatitis (SAP), one of the acute abdominals in the clinic, accounts for $10 \%$ to $20 \%$ of all acute pancreatitis cases (1). Rapid onset characterizes it, rapid progression, and poor prognosis (1). In addition, it causes inflammatory cascades and organ failure in its early stages, resulting in high mortality (1). Therefore, SAP is a serious threat to human health, life, and the economy (1). Despite extensive research and efforts to develop pharmacological tools, there are currently no specific treatments for SAP (1). Therefore, determining the pathophysiological mechanisms underlying SAP is crucial to identify effective therapeutic drugs and preventive measures for SAP.

The pathogenesis of SAP is complex and involves the abnormal activation of pancreatic inflammatory mediators, microcirculation dysfunction, translocation of intestinal flora, apoptosis, and oxidative stress. A large number of latest studies have shown that microcirculation disorder plays an extremely important role in the progression of SAP. It is not only the initiating factor of SAP, but also the factor of sustained and aggravated injury. Acute pancreatitis can damage the pancreas and systemic microcirculation, thus forming a vicious circle $(1,2)$. Additionally, the immune-inflammatory response-mediated pancreatic microcirculation dysfunction is always active, which is also an important molecular biomarker to determine the outcome of pancreatitis (2). In particular, the activation of the inflammatory immune response relies on the recruitment and migration of neutrophils in SAP (3). Therefore, it is an attractive treatment strategy to improve SAP by suppressing the dysfunction mediated by the inflammatory response of the pancreatic microcirculation by promoting neutrophil inactivation.

HMGB1, one of the endogenous molecules secreted from apoptotic and necrotic cells, has a pivotal influence on the development of SAP by activating the immune response (2). Once pancreatic cells are harmfully stimulated, HMGB1 in the nucleus will be modified by acetylation and then released to the extracellular space where it pairs with receptors (TLR-4) on the surface of macrophages to induce TLR-4-mediated immune responses $(2,4)$. This further promotes the release of HMGB1 and amplifies the immune reaction. It is worth noting that HMGB1TLR-4 signaling stimulates macrophages to secrete the pro-inflammatory cytokine IL-23 and IL-23, promoting Th17, $\gamma \delta$ T, NK, and NKT cells to secrete IL-17A to regulate neutrophil activation (5). In addition, the activity and nucleocytoplasmic shuttle of HMGB1 depend on the acetylation degree of HMGB1. SIRT1, one of the key acetylation modifying enzymes, can suppress HMGB1 transcription by deacetylation of HMGB1. Therefore, it is an effective strategy to alleviate the activation mediated by TLR-4 of a neutrophil by triggering SIRT1/HMGB1 signaling in SAP development (6).

Dachengqi decoction (DCQD) is a traditional decoction widely used for SAP treatment $(7,8)$. Its main ingredients include Houpu, Da Huang, Mangxiao, and Zhi Shi (7). DCQD appears positively associated with the improvement of pancreatic microcirculation in $\operatorname{SAP}(8,9)$. However, it is a pity that the concrete mechanism that DCQD regulates HMGB1 activation and microcirculation is not clear to date. Therefore, the purpose of the study was to confirm that the improvement of DCQD in pancreatic microcirculation is associated with the suppression of the neutrophil-mediated immune-inflammatory response. However, it promotes the inactivation of the HMGB1TLR-4-IL-23-IL-17A axis by targeting the SIRT1 signal pathway in SAP. We present the following article in accordance with the ARRIVE reporting checklist (available at https://dx.doi.org/10.21037/gs-21-655).

\section{Methods}

\section{Drugs, reagents, instruments, and animals}

DCQD consists of Houpu, Da Huang, Mangxiao, and Zhi Shi. The above herbs were acquired from Lv Yuan Pharmaceutical Co., Ltd. in Chengdu. The DCQ granules collected were dissolved in saline to obtain DCQD 
( $2 \mathrm{~g} / \mathrm{mL}$ ). According to the previous article, the concrete, specific compatibility, steps, extraction, and use (9).

Reagents were obtained from the following sources: sodium taurocholate, cerulean (Shanghai Dibao Biology Technology Co., Ltd.); lipase, and amylase, HMGB1, TNF- $\alpha$, IL-23, IL-1 $\beta$, KC, IL-17A, LIX, IL-6, and MIP2 ELISA Kits (Biyuntian); TLR-4, HMGB1 and AcetyHMGB1 antibodies (Abgent, USA); Myeloperoxidase (MPO) and CD68 Colorimetric Activity Assay Kit (BioVision, USA). EX527, r-HMGB1, neutralizing IL23p19, and IL-17A (Biolegend, San Diego, CA, USA) were purchased from Sigma.

The following instruments were purchased: enzyme labeling instrument, two-dimensional electrophoresis instrument, PCR (Bio-Rad, USA), paraffin embedding and pathological section machines (Leica, Germany), real-time PCR (Applied Biosystems, USA), Western blot imager (Pei Qing, Shang Hai).

Seventy healthy male SD rats (weighing $220 \pm 30$ g, 6-8 weeks) were obtained from Sichuan University. The rats were located in a standard SPF environment with humidity of $60 \% \pm 4 \%$ and a temperature of $22 \pm 2{ }^{\circ} \mathrm{C}$. All animal procedures were approved by the animal care committee of the Sichuan Provincial People's Hospital (No. 2011220), and the experimental protocols were strictly carried out based on NIH Guidelines for the care and use of animals. A protocol was prepared before the study without registration.

\section{Animal grouping, model establishment, and intervention}

The 70 rats were randomly separated into seven groups: Sham, SAP, DCQD, EX527, anti-IL-17, recombinant HMGB1(r-HMGB1), and anti-IL-23p19 groups ( $\mathrm{n}=10)$. Rats were fed ad libitum for 7 days before establishing the SAP model.

Rats in the SAP, DCQD, EX527, anti-IL-17, r-HMGB1, and anti-IL-23p19 groups were anesthetized with pentobarbital sodium $(40 \mathrm{mg} / \mathrm{kg})$. An upper abdominal incision was made by cutting open the skin and muscle to expose the bile duct, temporarily clamped to the small artery at the portal site on the liver. The insulin injection needle was stretched into the biliopancreatic duct by the nipple, and sodium taurocholate $(1 \mathrm{mg} /$ $\mathrm{kg}$ ) got into the biliopancreatic duct by injection. After $2 \mathrm{~h}$ of injecting sodium taurocholate, the animal in the EX527, DCQD, and r-HMGB1 group were treated with DCQD using an oral gavage at $20 \mathrm{~g} / \mathrm{kg}$ dose at $2 \mathrm{~h}$ after operation (9). Animals in the EX527 and r-HMGB1 group were also treated with EX527 $(10 \mathrm{mg} / \mathrm{kg})$ or r-HMGB1 $(100 \mu \mathrm{g} / \mathrm{kg})$ by intraperitoneal injection, according to the previous article $(10,11)$. Rats in the anti-IL-17A groups and the anti-IL-23p19 group were treated with neutralizing antibodies against IL-17A $(1 \mathrm{mg} / \mathrm{kg})$ and IL-23p19 $(0.2 \mathrm{mg} / \mathrm{kg})$, respectively, by intraperitoneal injection, according to the previous article $(12,13)$. After $12 \mathrm{~h}$, rats in all groups were sacrificed with pentobarbital sodium and serum, and pancreatic tissue was collected for further study.

\section{Cell culture}

Rat pancreatic acinar cell lines (AR42J) and RAW264.7 were acquired from ATCC (Rockville, MD, USA). After 30 min of treatment without or with DCQD, AR42J cells were co-incubated with cerulean for $24 \mathrm{~h}$.

\section{Gene silencing}

AR42J cells were transfected with control siRNA or TLR4. The RAW 264.7 cells were intervened with rHMGB1.

\section{TLR4 overexpression}

RAW 264.7 cells were designated to transfect TLR4 and control genes using Lipofectamine2000, according to the instructions. TLR4 and IL-23 expression were examined after transfection at $24 \mathrm{~h}$.

\section{Assaying cell viability}

MTT was used to evaluate cell survival, and the concrete steps were based on the instruction. AR42J cells were preconditioned with or without DCQD, EX527, or r-HMGB1 $(0.004 \mathrm{~g} / \mathrm{mL})$, successively 24 co-incubation with cerulean $(10 \mathrm{nmol} / \mathrm{L})$.

\section{Microcirculation of the pancreas}

Each rat was injected with $1.5 \mathrm{~mL}$ of FITC-RBC through the tail vein. We measured flow velocity, functional blood vessel number, red blood cell flow, and blood vessel number to evaluate pancreatic microcirculation using the BI-2000 medical image analysis system.

\section{Histology and wet/weight}

After performing hematoxylin and eosin (HE) staining, 
the excised pancreatic tissue was sectioned and mounted in paraffin, after performing hematoxylin and eosin (HE) staining to evaluate the pathological morphology of the pancreas. The pancreas was assayed under a light microscope. Pancreatic tissue was classified by evaluating necrosis + hemorrhage + edema + inflammation according to the modified Kusske scoring standard blinded by two independent pathologists (9). No inflammatory cell infiltration, hemorrhage, edema and necrosis were scored 0 ; necrosis area $(1-10 \%)$, hemorrhage and edema $(0-25 \%)$ were 1 point; necrosis area (11-20\%), hemorrhage and edema (25-50\%) were 2 points; necrosis area (21-30\%), hemorrhage and edema (50-75\%) were 3 points; necrosis area $(>30 \%)$ hemorrhage and edema $(50-55 \%)$ were 4 points; 0.5 point for 5 inflammatory cells and 4 points for more than 30 inflammatory cells. The weighted index of the pancreas was calculated as the weight of the pancreas (g)/ body weight $(\mathrm{g}) \times 100 \%$.

\section{Amylase and lipase activity assays in serum}

The blood collected was centrifuged to obtain the supernatant. The amylase and lipase activity was assayed by exploiting colorimetric activity kits for amylase and lipase, following the manufacturer's instructions.

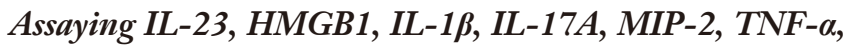 LIX, IL-6, and KC}

IL-23, HMGB1, IL-1 $\beta$, IL-17A, MIP-2, TNF- $\alpha$, LIX, IL6 , and $\mathrm{KC}$ concentrations in the pancreas and serum were determined using rat ELISA kits for each protein, following the manufacturer's instructions.

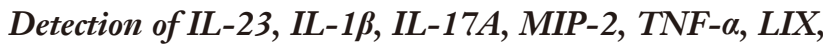 $I L-6$, and KC $m R N A$ expression in the pancreas}

Total RNA was extracted using the TRIzol method after homogenizing the frozen pancreas in liquid nitrogen. Realtime PCR was exploited to detect mRNA expression. The concrete steps were in line with the instructions. The primer sequences are displayed in Table 1 .

\section{Examining SIRT1 activity}

SIRT1 activity was evaluated by the Fluorescent Assay Kit for SIRT1 activity based on instructions.

\section{Western blotting}

Total, nuclear, and cytoplasmic proteins were extracted from pancreatic tissues by lysis, centrifugation, and quantification. Western blot analysis was performed using SIRT1 (1:400), Acey-HMGB1 (1:400), TLR-4 (1:1,000), HMGB1 (1:400), $\beta$-actin $(1: 4,000)$ and Histone $(1: 1,000)$. Signals were detected by incubation with a secondary antibody, followed by exposure to the ECL-Plus reagent.

\section{MPO and CD68 assay}

Immunohistochemistry was used to evaluate the expression of MPO and CD68 in pancreatic tissues.

\section{Statistical methods}

All documents are displayed as mean \pm SEM. T-test and one-way ANOVA tests were used to assay multiple comparisons. Tukey's post-hoc test was exploited to evaluate individual means. $\mathrm{P}<0.05$ was considered significant.

\section{Results}

Wet/weight of the pancreas, serum amylase and lipase activity, cell viability, and survival rates

Compared with the SAP group, the wet/weight, activity of amylase and lipase, and mortality in the Sham, DCQD, anti-IL-17A, and anti-IL-23-p19 groups were obliviously decreased $(\mathrm{P}<0.05)$. However, with the DCQD group, the wet/weight, activity of amylase and lipase, and the mortality in the markedly increased $(\mathrm{P}<0.05)$ (Figure 1A-1C).

AR42J cells were treated with Cerulean to simulate SAP in rats. Figure $1 C, 1 D$ shows that cell viability in the Sham, anti-IL-17A, DCQD, and anti-IL-23-p19 groups was significantly up-regulated $(\mathrm{P}<0.05)$. Nevertheless, with the DCQD group, cell viability in the EX527 and r-HMGB1 groups was markedly up-regulated $(\mathrm{P}<0.05)$ (Figure 1D,1E) $(\mathrm{P}<0.05)$.

\section{Pancreatic histopathological morphology and the expression of CD 68 and MPO in the pancreas}

HE and immunohistochemistry were used to assess pancreatic histological morphology and CD 68 and MPO expression, respectively. The Sham group showed that the pancreas of some rats had slight edema, a small amount of 
Table 1 The primer for IL-23, IL-17A, KC, LIX, MIP-2, IL-6, IL-1 $\beta$, and TNF- $\alpha$

\begin{tabular}{lll}
\hline Gene & Sense group $\left(5^{\prime}-3^{\prime}\right)$ & Antisense group $\left(5^{\prime}-3^{\prime}\right)$ \\
\hline HMGB1 & ATGGGCAAAGGAGTC & ATTCATCATCATCATCTTCT \\
IL-17A 23 & GGAAAGTGGAC-CACCACA & CACACCCACCAGCATCTTCTC \\
KC & AGGACTTGTGCTGTTCTTGTTTTG & CTCTGGGGTTGTTTCTTTCTCTT \\
LIX & TCACGCTTCTGGGCCTGTTG & CAGCCGACTCATTGGGATCATC \\
$M I P-2$ & TCACGCTTCTGGGCCTGTTG & CAGCCGACTCATTGGGATCATC \\
$I L-6$ & GGCAAGGCTAACAGACCTGGAAAG & CACATCATCAGGTACGATCCAGGCTTC \\
$I L-1 \beta$ & TGCGCTGGGCTTAGATCATT & TGGATGCCTTTTATGTCGTCT \\
$T N F-\alpha$ & AGGGAAATCGTGCGTGACAT & GAACCGCTCATTGCCGATAG \\
$\beta$-actin & ACCAAGGATGAGGGCGACTA & CAGGCTTATGCCACCACACTT \\
\hline
\end{tabular}

inflammatory infiltration, but no necrosis or hemorrhage. Pancreatic tissues of the SAP group had severely damaged pancreatic tissue, widespread edema and hemorrhage, a large number of inflammatory cell infiltration, and necrosis of acinar cells with pathological injury scores higher than those of the Sham group $(\mathrm{P}<0.05)$. Rat pancreatic tissues in the anti-IL-23-p19, DCQD, and anti-IL-17A groups had edema slightly, a small amount of inflammatory infiltration, hemorrhage, and necrosis with lower pathological injury scores than in the SAP group $(\mathrm{P}<0.05)$. Yet with the DCQD group, the r-HMGB1 and EX527 groups had more pancreatic tissue necrosis, inflammatory cell infiltration, edema, and hemorrhage with higher pathological injury scores $(\mathrm{P}<0.05)$ (Figure 2).

Compared to the sham group, the SAP group has a higher expression of CD68 and MPO $(\mathrm{P}<0.05)$. Compared to the SAP group, the DCQD group, the anti-IL-17A, and anti-IL-23-p19 groups have lower expressions of CD68 and MPO $(\mathrm{P}<0.05)$. Compared to the DCQD group, EX527 and r-HMGB1 have higher expressions of CD68 and MPO $(\mathrm{P}<0.05)$ (Figure 2).

\section{Microcirculatory function of the pancreas}

Impaired pancreas microcirculation is a typical clinical manifestation of severe pancreatitis $(2,3)$. Compared to the sham group, the SAP group has a lower blood flow velocity, the number of functional blood vessels, $\mathrm{RBC}$ flow, and the number of blood vessels $(\mathrm{P}<0.05)$. Compared to the SAP group, the DCQD, anti-IL-17A, and anti-IL-23-p19 groups had blood flow velocity, number of the functional blood vessels, RBC flow, and number of blood vessels $(\mathrm{P}<0.05)$. Furthermore, the r-HMGB1 and EX527 groups had a higher blood flow velocity, the number of functional blood vessels, the flow of RBC, and the number of blood vessels than the DCQD group $(\mathrm{P}<0.05)$ (Figure 3).

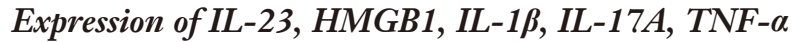 and IL-6}

The immune-inflammatory response plays a key role in the progression of SAP $(2,3)$. Therefore, the study examined the level of IL-23, HMGB1, IL-1 $\beta$, IL-17A, TNF- $\alpha$, and IL-6 in serum and pancreas at both gene and protein levels. The SAP group has a higher level of HMGB1, IL-17A, IL-23, IL-6, TNF- $\alpha$, and IL-1 $\beta$ in serum and pancreases than the Sham, DCQD, anti-IL-17A, and anti-IL-23-p19 groups $(\mathrm{P}<0.05)$. Furthermore, the EX527 and r-HMGB1 groups have higher levels of IL-23, HMGB1, IL-1 $\beta$, IL$17 \mathrm{~A}, \mathrm{TNF}-\alpha$, and IL- 6 in serum and pancreas at gene and protein levels compared to the DCQD group $(\mathrm{P}<0.05)$ (Figure 4).

\section{Neutrophil activation}

MPO is a surface marker of neutrophils, and MIP-2, LIX, and $\mathrm{KC}$ are neutrophil chemokines that reflect neutrophil activation (14). Therefore, we measured the pancreatic MPO activity and the protein and mRNA levels of MIP2, LIX, and KC in serum and pancreas. The SAP group has higher pancreatic MPO activity and more MIP-2, LIX, and $\mathrm{KC}$ expression than the sham group, DCQD, anti-IL- 

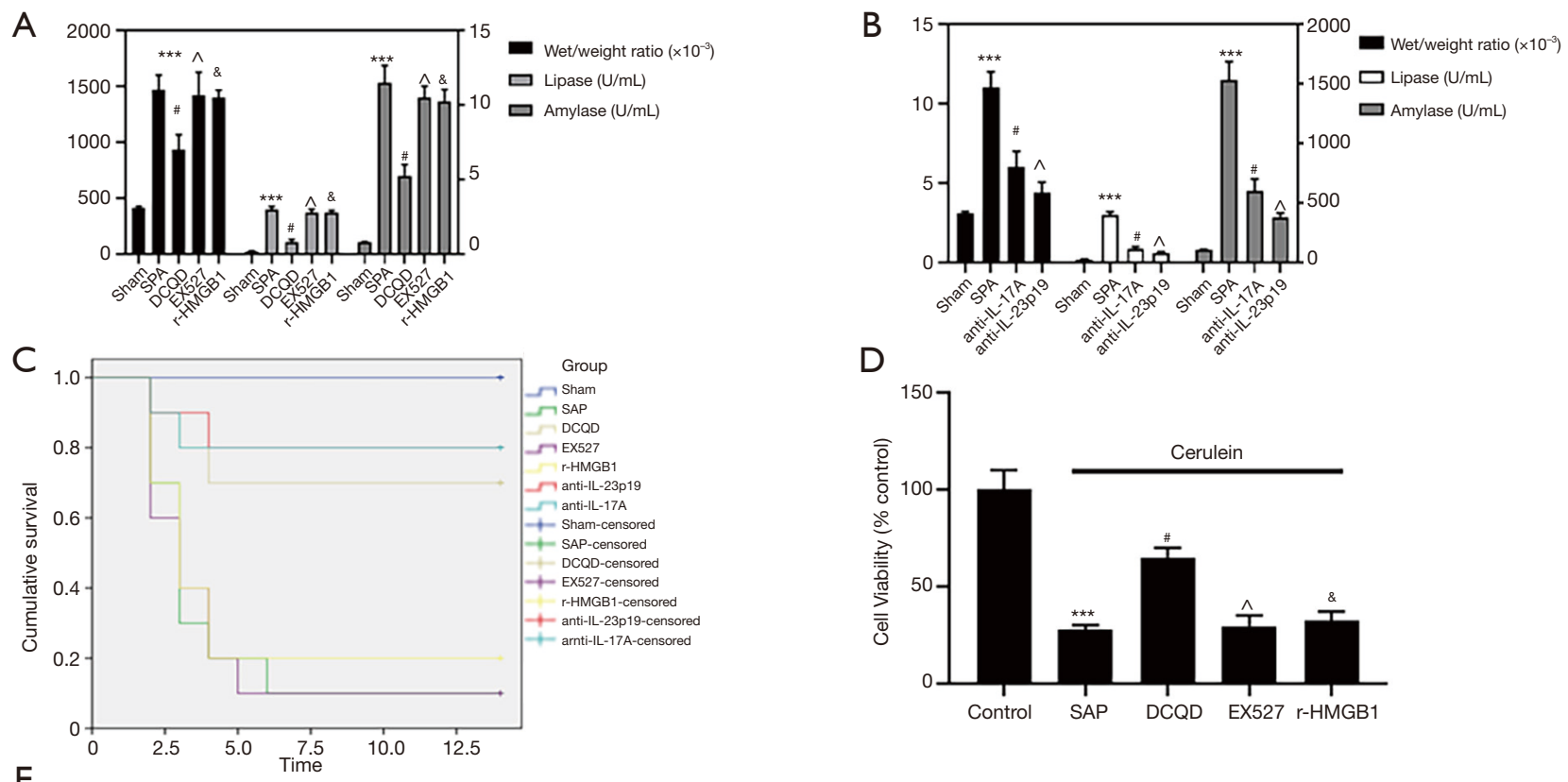

E

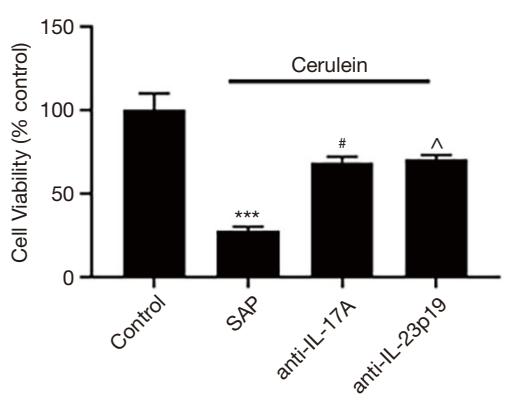

Figure 1 The effect of DCQD, EX527, anti-IL-17A, r-HMGB1, and anti-IL-23p19 on wet/weight, amylase and lipase activity, surviving rats, and AR42J cell viability in SAP. (A) The influence of DCQD, EX527 and r-HMGB1 on wet/weight, amylase and lipase activity. (B) The influence of anti-IL-17A and anti-IL-23p19 on wet/weight, amylase and lipase activity. (C) The influence of DCQD, EX527, anti-IL17A, r-HMGB1 and anti-IL-23p19 on the survive rats in SAP. (D) The effect of DCQD, EX527 and anti-IL-17A, r-HMGB1and anti-IL23 p19 on the AR42J cell viability in SAP. (E) The effect of anti-IL-17A and anti-IL-23p19 on the AR42J cell viability in SAP; ***, P<0.001 (SAP vs. Sham); ", $\mathrm{P}<0.05$ (SAP vs. DCQD); ^ $\mathrm{P}<0.05$ (EX527 vs. DCQD); ${ }^{\star}, \mathrm{P}<0.05$ (r-HMGB1 vs. DCQD); ^, $\mathrm{P}<0.05$ (anti-IL-23p19 vs. SAP);, $\mathrm{P}<0.05$ (anti-IL-17A vs. SAP); ${ }^{* * *}, \mathrm{P}<0.001$ (SAP vs. Control). DCQD, Dachengqi decoction; SAP, severe acute pancreatitis.

17A, and anti-IL-23-p19 groups $(\mathrm{P}<0.05)$. Furthermore, the r-HMGB1 and EX527 groups have higher pancreatic MPO activity and more expression of MIP-2, LIX, and KC compared to the DCQD group $(\mathrm{P}<0.05)$ (Figure 5).

\section{The SIRT1, acety-HMGB1 and TLR-4 expression,} HMGB1 translocation, and SIRT1-HMGB1 combination in the pancreas

The HMGB1-TLR4 signal pathway significantly influences
SAP, and SIRT1 regulates HMGB1 can activation through deacetylation $(7,8)$. Therefore, we examined the activity of SIRT1, the combination of SIRT1 and HMGB1, and the level of SIRT1, acety-HMGB1, TLR4, and the mitigation of HMGB1 from nuclear to the cytoplasm in vitro and in vivo. The SAP group had a higher expression of acetyHMGB1, TLR-4, and cytoplasmic HMGB1 than the sham group with lower SIRT1 activity, lower level of nuclear HMGB1, SIRT1, and lower combination of SIRT1HMGB1 $(\mathrm{P}<0.05)$, while the DCQD group showed an 

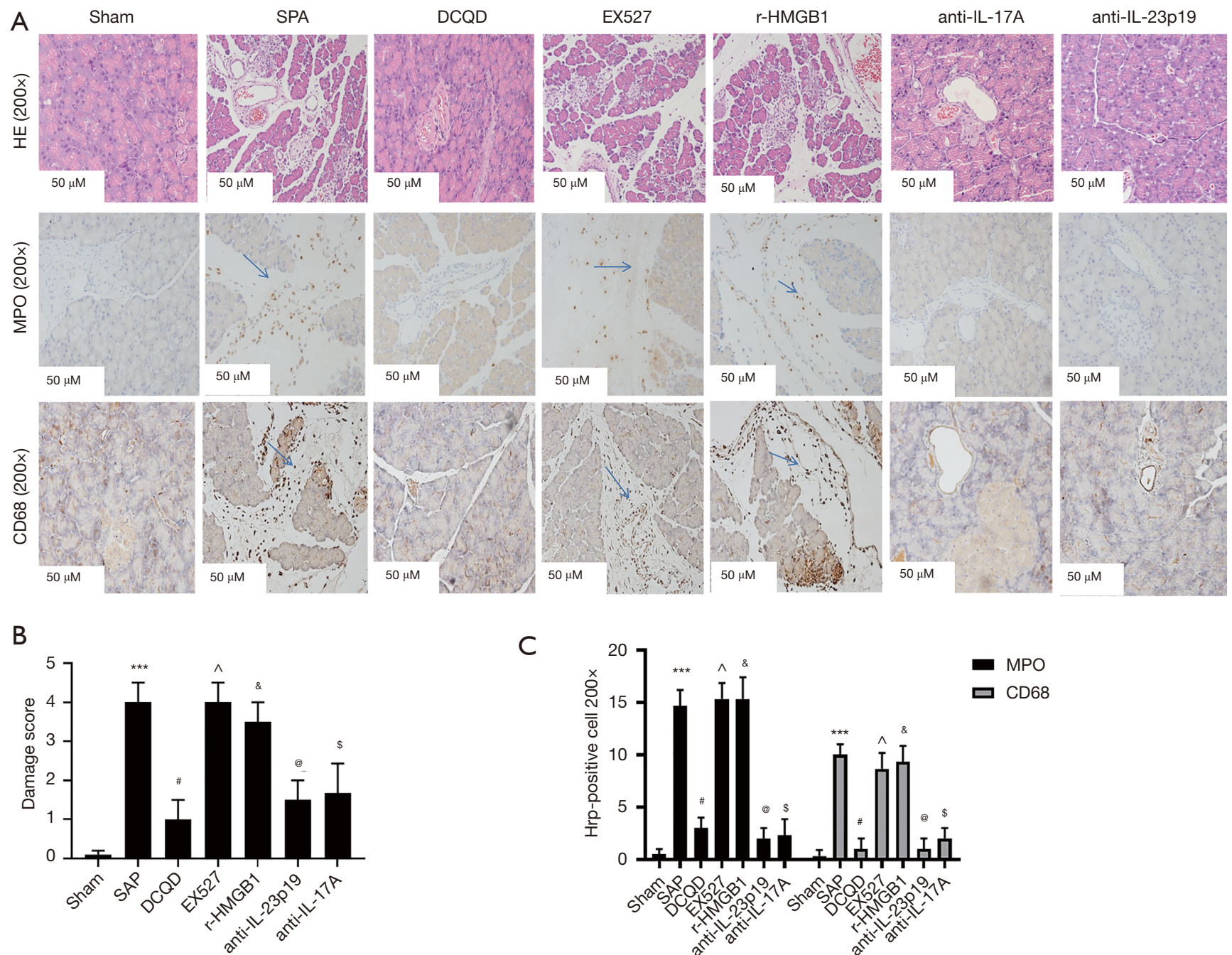

C

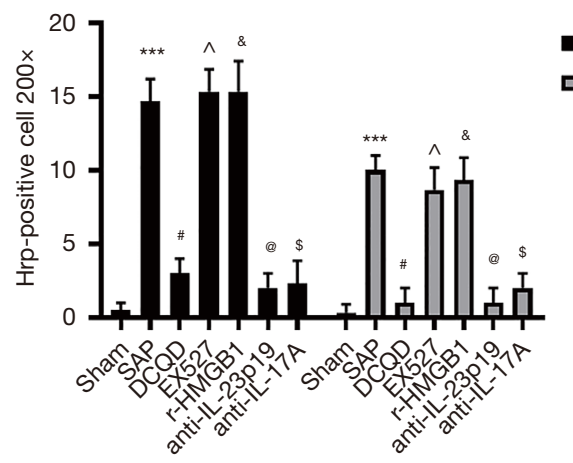

Figure 2 The influence of DCQD, EX527, anti-IL-17A, r-HMGB1, and anti-IL-23p19 on pancreatic histopathological morphology, and CD68 and MPO expression in SAP. (A) Representative results from HE stains (magnification $\times 200$ ) on the histopathological morphology of the pancreas and immunohistochemistry for CD68 and MPO; (B) the damage score was evaluated by semiquantitative analysis. (C) CD68 and MPO expression were evaluated by semiquantitative analysis in SAP. ***, $\mathrm{P}<0.001$ (SAP vs. Sham); ${ }^{*}, \mathrm{P}<0.05$ (SAP vs. DCQD); , $\mathrm{P}<0.05$ (EX527 vs. DCQD); ${ }^{\&}, \mathrm{P}<0.05$ (r-HMGB1 vs. DCQD); ${ }^{\circledR}, \mathrm{P}<0.05$ (anti-IL-23p19 vs. SAP); $\$, \mathrm{P}<0.05$ (anti-IL-23p19 vs. SAP). The blue arrows indicate the positive place. DCQD, Dachengqi decoction; SAP, severe acute pancreatitis.

increase in SIRT1 activity, a combination of SIRT1 and HMGB1, and a level of SIRT1 and nuclear HMGB1 with a decrease in the level of acety-HMGB1, TLR-4, and cytoplasmic HMGB1 than the SAP group $(\mathrm{P}<0.05)$. Furthermore, the EX527 group showed a higher level of acety-HMGB1, TLR-4, and HMGB1 in the cytoplasm than the DCQD group with a lower combination of SIRT1HMGB1, lower expression of SIRT1 and nuclear HMGB1, and lower activity of SIRT1 $(\mathrm{P}<0.05)$ (Figure 6).

\section{The release of $I L-23$, though relying on the HMGB1/ TLR-4 way in SAP}

As shown in Figure 7A,7B, r-HMGB1 up-regulated IL23 secretion and increased the IL-23 and TLR-4 levels $(\mathrm{P}<0.5)$. Compared to the SAP group, the DCQD and TLR-4 siRNA groups have lower IL-23 and TLR-4. However, overexpression of TLR-4 can up-regulate IL-23 and TLR-4 expression using a TLR-4 expression plasmid, 


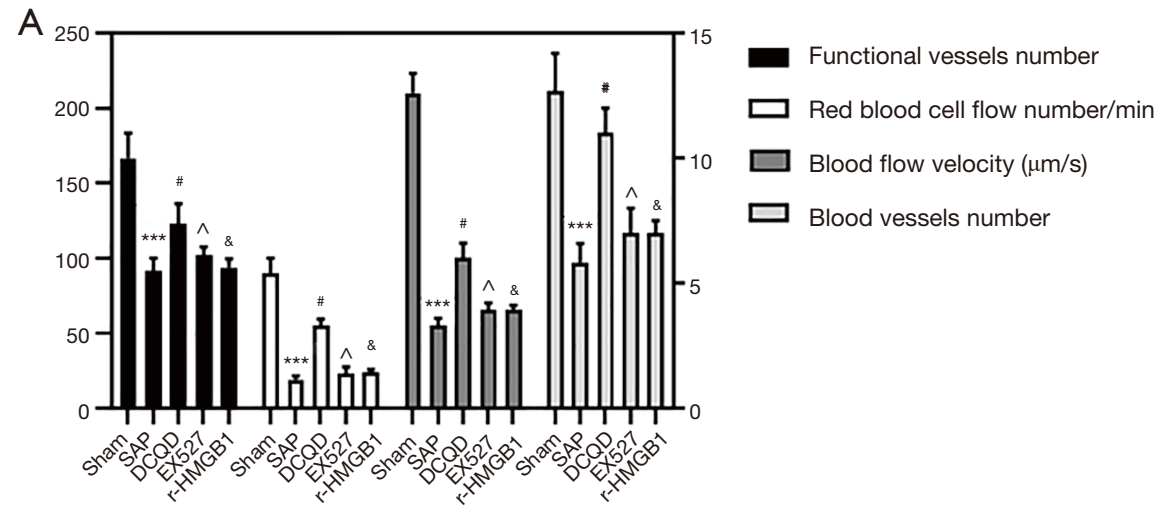

B

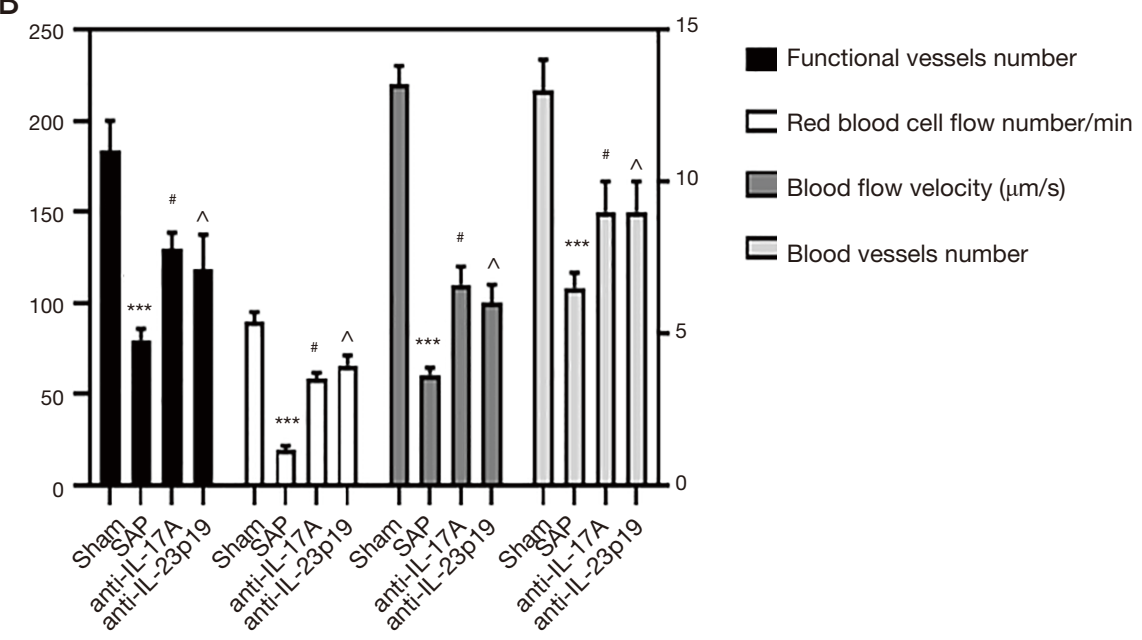

Figure 3 The effect of DCQD, EX527, anti-IL-17A, r-HMGB1, and anti-IL-23p19 on pancreatic microcirculatory function in SAP. (A) The effect of DCQD, EX527, and r-HMGB1 on blood flow velocity, number of functional blood vessel, RBC flow and number of blood vessel. (B) The influence of anti-IL-17A and anti-IL-23p19 on the blood flow velocity, number of functional blood vessel, RBC flow and number of blood vessel. ***, $\mathrm{P}<0.001$ (SAP vs. Sham); ${ }^{*}, \mathrm{P}<0.05$ (SAP vs. DCQD);, $\mathrm{P}<0.05$ (EX527 vs. DCQD); ${ }^{*}, \mathrm{P}<0.05$ (r-HMGB1 vs. DCQD); ^, $\mathrm{P}<0.05$ (anti-IL-23p19 vs. SAP); * $\mathrm{P}<0.05$ (anti-IL-17A vs. SAP). DCQD, Dachengqi decoction; SAP, severe acute pancreatitis.

demonstrating higher expression of IL-23 and TLR-4 in the TLR-4 plasmid group than the SAP group $(\mathrm{P}<0.05)$.

\section{The DCQD signal patbway in the pancreatic microcirculatory system in SAP (Figure 8)}

DCQD treatment improves SAP-induced pancreatic microcirculatory dysfunction by inhibiting neutrophilmediated inflammation via inactivating HMGB1-TLR-4IL-23-IL-17A signaling by targeting SIRT1.

\section{Discussion}

One common clinical critical illness, SAP, easily caused multiple organ failure and frequent death (1). This study used sodium taurodeoxycholate or cerulean to induce $\mathrm{SAP}$ in vivo and in vitro, regardless. In vivo, SAP caused a disturbance in the microcirculation of the pancreas, an increase in serum amylase and lipase activity, and an increase in the wet weight ratio of the pancreas. In vitro, cerulean can significantly decrease AR42J cell viability, which is consistent with earlier studies (9). These indicated that SAP 


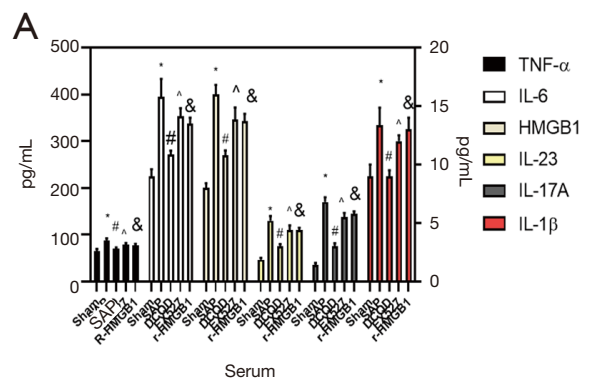

B
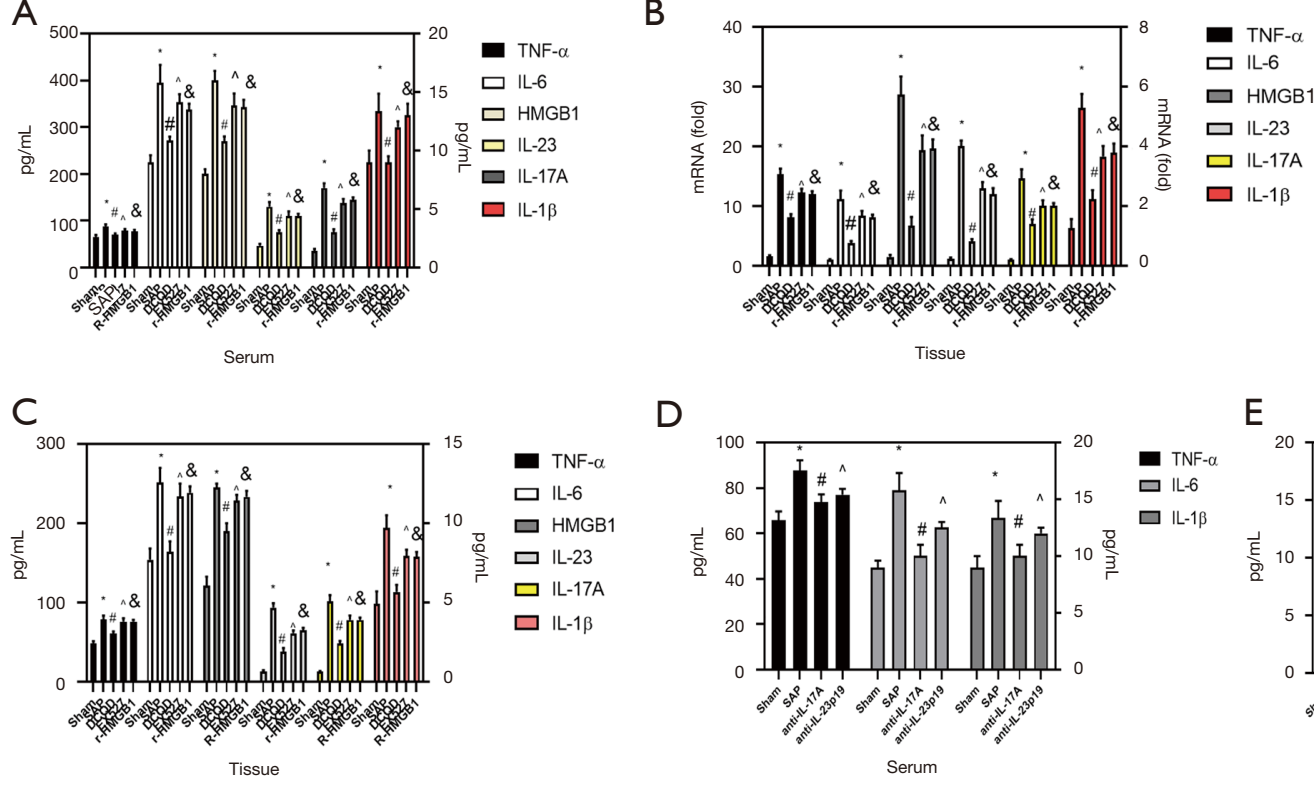

D

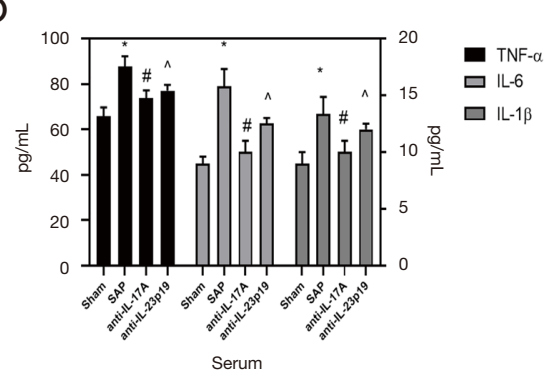

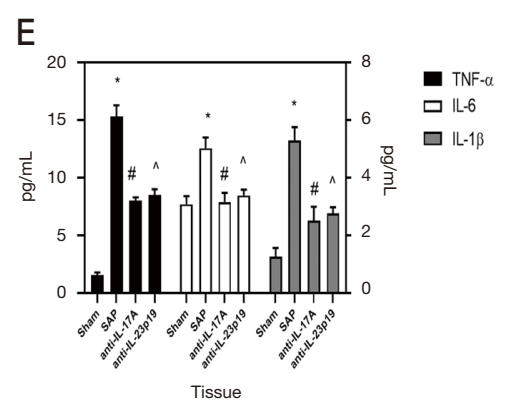

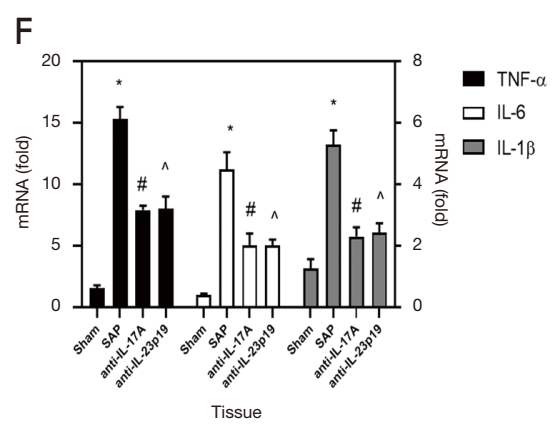

G

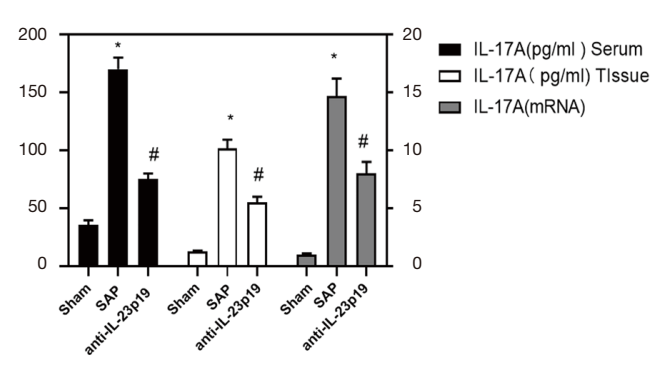

Figure 4 The influence of DCQD, EX527, anti-IL-17A, r-HMGB1, and anti-IL-23p19 on the level of IL-23, HMGB1, IL-1 $\beta$, IL17A, TNF- $\alpha$, and IL-6 in SAP. (A) ELISA was used to assess the influence of DCQD, EX527 and r-HMGB1 on the serum level of IL23, HMGB1, IL-1 $\beta$, IL-17A, TNF- $\alpha$ and IL-6 in SAP; (B) RT-PCR was used to assess the effect of DCQD, EX527 and r-HMGB1 on the pancreatic mRNA of IL-23, HMGB1, IL-1 $\beta$, IL-17A, TNF- $\alpha$ and IL-6 in SAP; (C) ELISA was used to examine the effect of DCQD, EX527, and r-HMGB1 on the pancreatic level of IL-23, HMGB1, IL-1 $\beta$, IL-17A, TNF- $\alpha$ and IL-6 in SAP; (D) the ELISA was used to test the influence of anti-IL-17A and anti-IL-23p19 on the serum level of TNF- $\alpha$, IL-6 and IL-1 $\beta$ in SAP; (E) ELISA was used to measure the influence of anti-IL-17A and anti-IL-23p19 on the pancreatic level of TNF- $\alpha$, IL-6 and IL-1 $\beta$ in SAP; (F) RT-PCR was used to measure the influence of anti-IL-17A and anti-IL-23p19 on pancreatic mRNA of TNF- $\alpha$, IL-6 and IL-1 $\beta$ in SAP; (G) ELISA and RT-PCR were used to evaluate the influence of anti-IL-23p19 on the level of IL-17A in SAP. *, $\mathrm{P}<0.05$ (SAP vs. Sham); ${ }^{2}, \mathrm{P}<0.05$ (SAP vs. DCQD); ^, P<0.05 (EX527 vs. DCQD); ${ }^{\&}, \mathrm{P}<0.05$ (r-HMGB1 vs. DCQD); ^, $\mathrm{P}<0.05$ (anti-IL-23p19 vs. SAP); ${ }^{*}, \mathrm{P}<0.05$ (anti-IL-17A vs. SAP). DCQD, Dachengqi decoction; SAP, severe acute pancreatitis.

in vivo and in vitro models was successfully established. We also showed that DCQD, anti-IL-17A, or anti-IL-23-p19 treatment remarkably decreased lipase and amylase activity, reduced microcirculatory pancreatic pathological injury and dysfunction, downregulated the weight index, and improved cerulean -induced the decline in the cell viability of AR42J.

Furthermore, both r-HMGB1 (an HMGB1 agonist) and
EX527 (SIRT1 inhibitor) effectively abated the protective effect of DCQD in SAP. The findings were supported, as they showed a higher serum activity of lipase and amylase, more serious pancreatic pathological injury, weight index and cell viability, and worse microcirculatory function in the EX527 and r-HMGB1 groups than in the DCQD group. Furthermore, previous studies have confirmed 
A

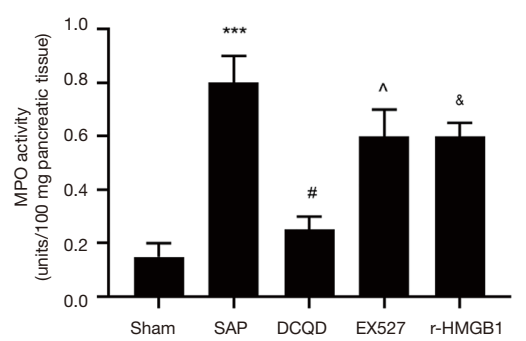

B

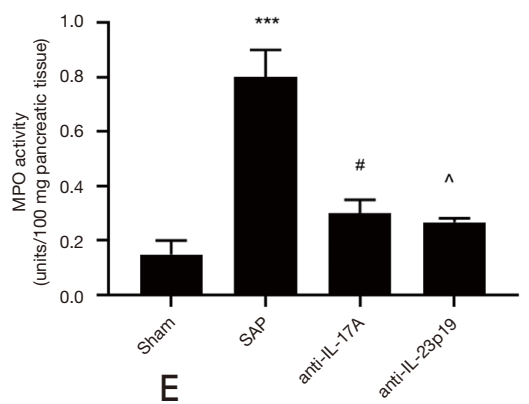

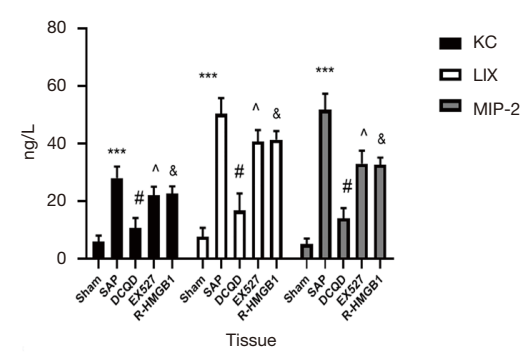

G

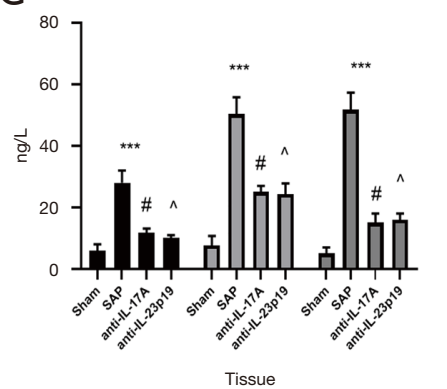

D

$\mathrm{H}$

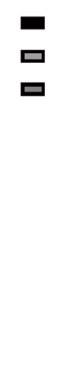

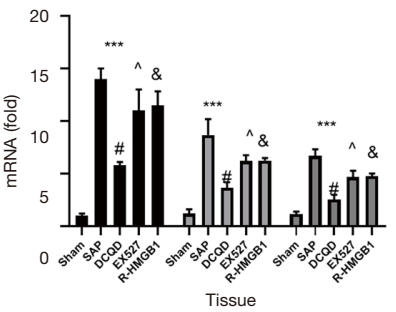

ש $\mathrm{KC}$

MIP-2
F

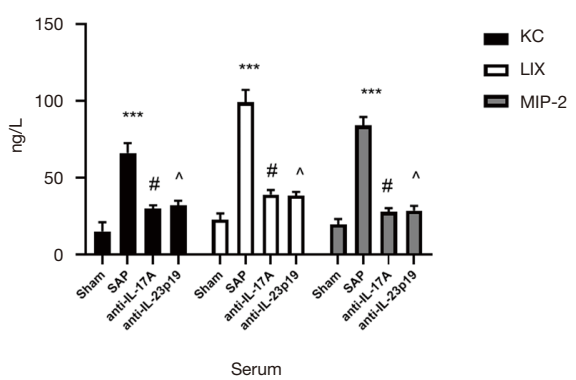

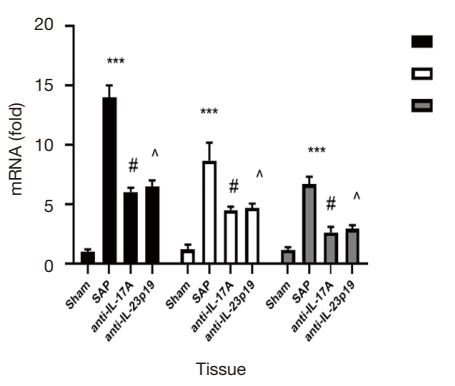

Figure 5 The influence of DCQD, EX527, anti-IL-17A, r-HMGB1 and anti-IL-23p19 on MPO activity and expression of MIP-2, KC, and LIX in SAP. (A) ELISA was exploited to evaluate the influence of DCQD, r-HMGB1, and EX527 on MPO activity in SAP; (B) ELISA was used to assess the influence of anti-IL-17A and anti-IL-23p19 on MPO activity in SAP; (C) ELISA was used to analyze the influence of DCQD, r-HMGB1, and EX527 on serum MIP-2, KC, and LIX level in SAP; (D) ELISA was exploited to evaluate the influence of DCQD, r-HMGB1, and EX527 on MIP-2, KC and LIX expression in SAP; (E) RT-PCR was exploited to measure the influence of DCQD, r-HMGB1 and EX527 on the MIP-2, KC and LIX mRNA expression in SAP; (F) ELISA was designated to measure the influence of anti-IL-17A and anti-IL-23p19 on the MIP-2, KC and LIX serum expression in SAP; (G) ELISA was designated to evaluate the influence of anti-IL-17A and anti-IL-23p19 on the MIP-2, KC and LIX pancreatic expression in SAP; (H) RT-PCR was used to evaluate the influence of anti-IL-17A and anti-IL-23p19 on the MIP-2, KC, and LIX pancreatic mRNA in SAP; ***, $\mathrm{P}<0.001$ (SAP vs. Sham); ${ }^{* * *}, \mathrm{P}<0.001$ (SAP vs. Control); ${ }^{*}, \mathrm{P}<0.05$ (SAP vs. DCQD); ^, $\mathrm{P}<0.05$ (EX527 vs. DCQD); ${ }^{\circledR}, \mathrm{P}<0.05$ (r-HMGB1 vs. DCQD); ^, $\mathrm{P}<0.05$ (anti-IL-23p19 vs. SAP);, $\mathrm{P}<0.05$ (anti-IL-17A vs. SAP). DCQD, Dachengqi decoction; SAP, severe acute pancreatitis; MPO, myeloperoxidase.

that activating the HMGB1 and IL-23/IL-17A pathways significantly aggravated pancreatic microcirculatory impairment. Therefore, our study further confirms that the protective effect DCQD on SAP was achieved through down-regulation of HMGB1-mediated pancreatic microcirculatory dysfunction, and the IL-23/IL-17A signal pathway plays an axis in regulating microcirculatory dysfunction in SAP.
Increasing evidence showed that the inflammatory response significantly influences TNF- $\alpha$, IL-6, and IL- $1 \beta$ are the most important symbol of inflammatory cytokines in SAP (14). TNF- $\alpha$ is an inflammation-initiating factor that can mediate the release of IL-6 and other inflammatory cytokines, stimulate oxygen free radicals and nitric oxide production, promote leukocyte chemotaxis and adhesion, and damage pancreatic tissue (15). IL-6 has a critical 

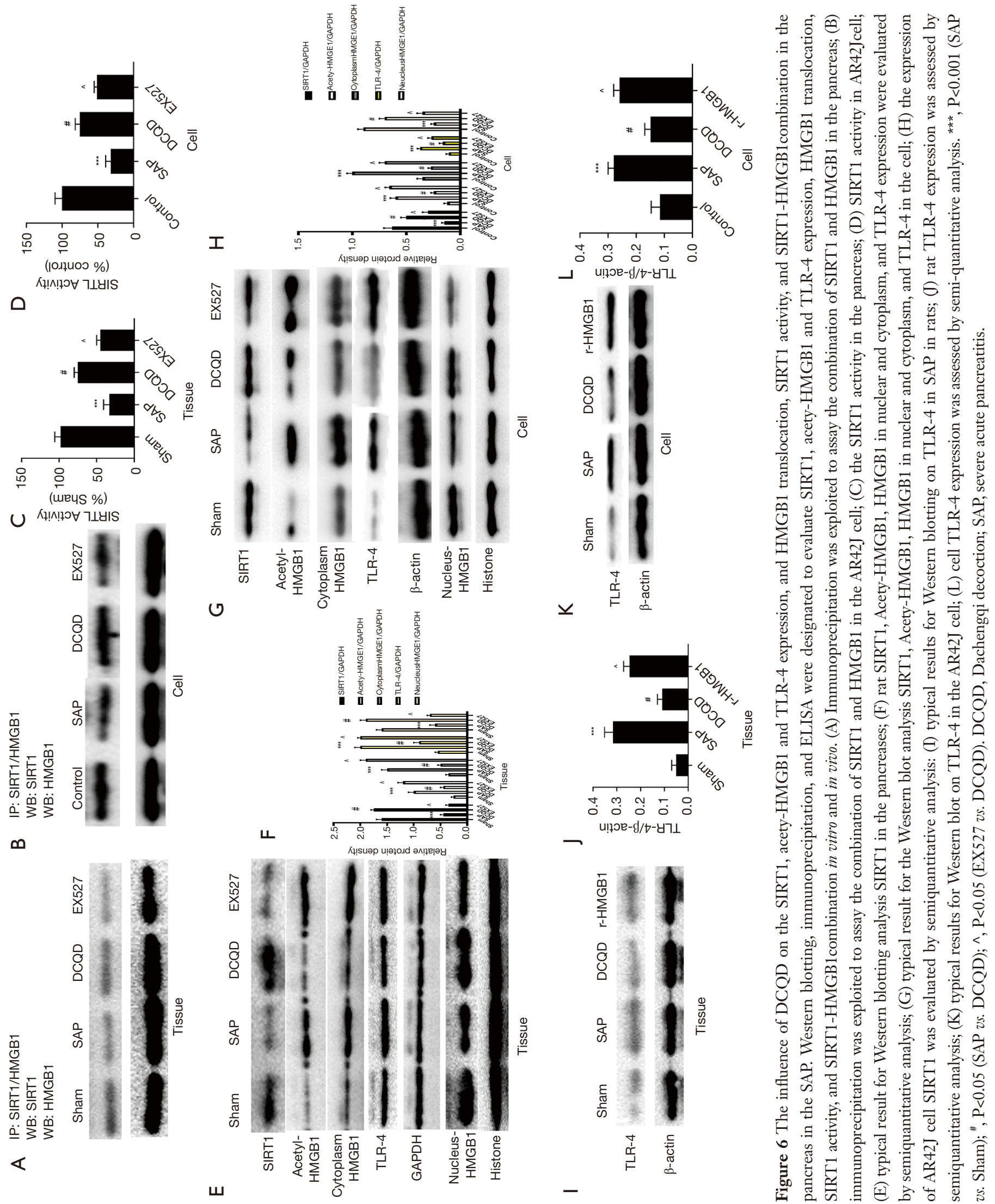


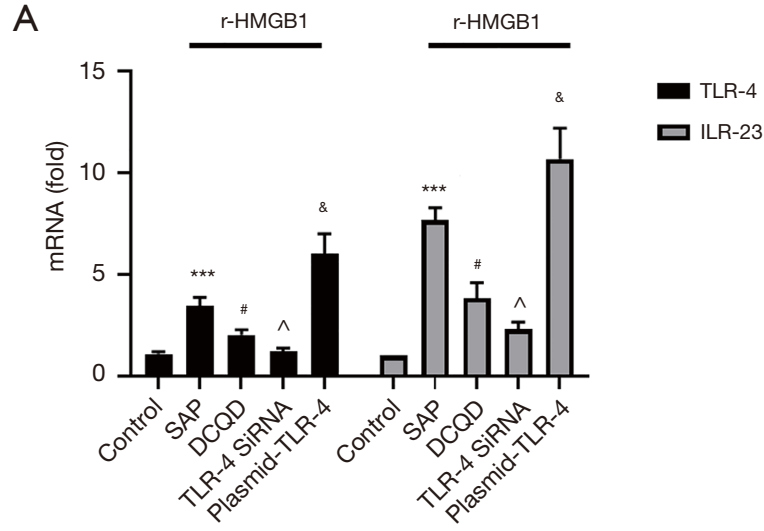

RAW264.7

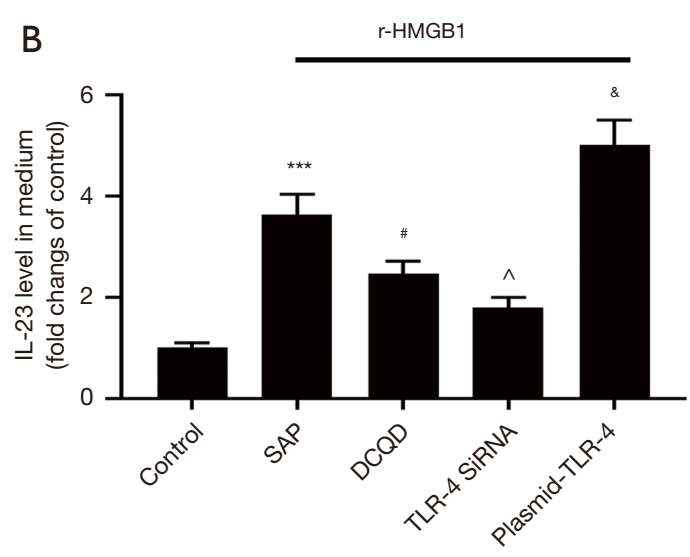

RAW264.7

Figure 7 The influence of DCQD on the expression of TLR-4 and IL-23 in SAP. (A) The influence of DCQD on the level of TLR4 and IL-23 mRNA in RAW264.7 was detected through RT-PCR; (B) ELISA was designated to evaluate the influence of r-HMGB1 on IL-23 expression in the supernatant in SAP. ${ }^{* *}, \mathrm{P}<0.001$ (SAP vs. Control); ", $\mathrm{P}<0.001$, (DCQD vs. SAP); ${ }^{\wedge}, \mathrm{P}<0.05$ (DCQD vs. siRNA-TLR-4); ${ }^{*}, \mathrm{P}<0.05$ (DCQD vs. plasmid-TLR-4). DCQD, Dachengqi decoction; SAP, severe acute pancreatitis.

influence in initiating an immune response in SAP, and is released primarily by macrophages, $\mathrm{T}$ lymphocytes, dendritic cells, and T cells $(14,15)$. IL-1 $\beta$ is deeply involved in the destruction of pancreatic tissue and the formation of edema in SAP (15). In this study, the intervention with antiIL-17A, DCQD, or anti-IL-23p19 significantly decreased TNF- $\alpha$, IL-6, and IL-1 $\beta$ expression. However, r-HMGB1 and EX527 can eliminate the influence of DCQD on the inflammatory response in SAP, as confirmed higher expression of TNF- $\alpha$, IL- 6 , and IL- $1 \beta$ in the r-HMGB1 and EX527 groups than in the DCQD group. The above results implied that DCQD could reduce SAP-induced microcirculatory dysfunction by inhibiting the HMGB1mediated inflammatory response, and the IL-23/IL-17A signal pathway can improve microcirculatory dysfunction by attenuating inflammation in SAP.

Interestingly, blocking neutrophil activation has previously been demonstrated to suppress the inflammatory response in SAP (7). Therefore, the intensity of the inflammatory response depends on the activation of neutrophils in SAP. In addition, MPO is a surface marker of neutrophils, and KC, LIX, and MIP-2 are neutrophil chemokines that reflect neutrophil activation by recruitment and migration of neutrophils. Treatment with anti-IL23-p19, DCQD, or anti-IL-17A decreased pancreatic MPO expression, activity, and MIP-2., KC, and LIX. However, the r-HMGB1 intervention can reduce the negative influence of DCQD on neutrophil inactivation and lessen neutrophil chemokines, as it showed higher MPO activity and higher expression of MIP-2, KC, and LIX in the r-HMGB1 group than in the DCQD group. These findings further demonstrated that anti-IL-23-p19, DCQD, or antiIL-17A reduce the inflammatory response by activating neutrophils in SAP.

IL-17A is a common molecule that critically modulates host defense against detrimental inflammatory stimulation (6). Controlling neutrophil activation and recruitment to the pancreas is the main mechanism of IL$17 \mathrm{~A}$ activity (7). IL-17A is mainly released from NKT cells, $\gamma \delta \mathrm{T}$ cells, Th17 cells, etc. $(6,15)$. In particular, the release of IL-17A is controlled by the heterodimeric cytokine IL-23 through the following mechanism $(16,17)$ : IL-23 triggers naïve $\mathrm{CD} 4+\mathrm{T}$ cells differentiation into Th17 and subsequently stimulates NKT cells to release IL-17A, along with anti-CD3. Furthermore, IL-23 promotes the release of IL-17A from $\gamma \delta$ T cells by working with IL-1 (17). In our study, SAP can stimulate the activation of IL-23/IL-17A signaling, and DCQD significantly promoted inactivation of the IL-23/IL-17A axis, as it showed a lower level of IL$17 \mathrm{~A}$ and IL-23 in the DCQD group than in the SAP group. However, r-HMGB1 can boost activating IL-23/IL-17A signaling in SAP, demonstrating higher IL-17A and IL23 levels in an r-HMGB1 group than the DCQD group. Furthermore, we also demonstrated that neutralizing IL23 reduced the expression of IL-17A in SAP, which further confirmed that IL-23 could promote IL-17A secretion. These results were in accordance with previous research 

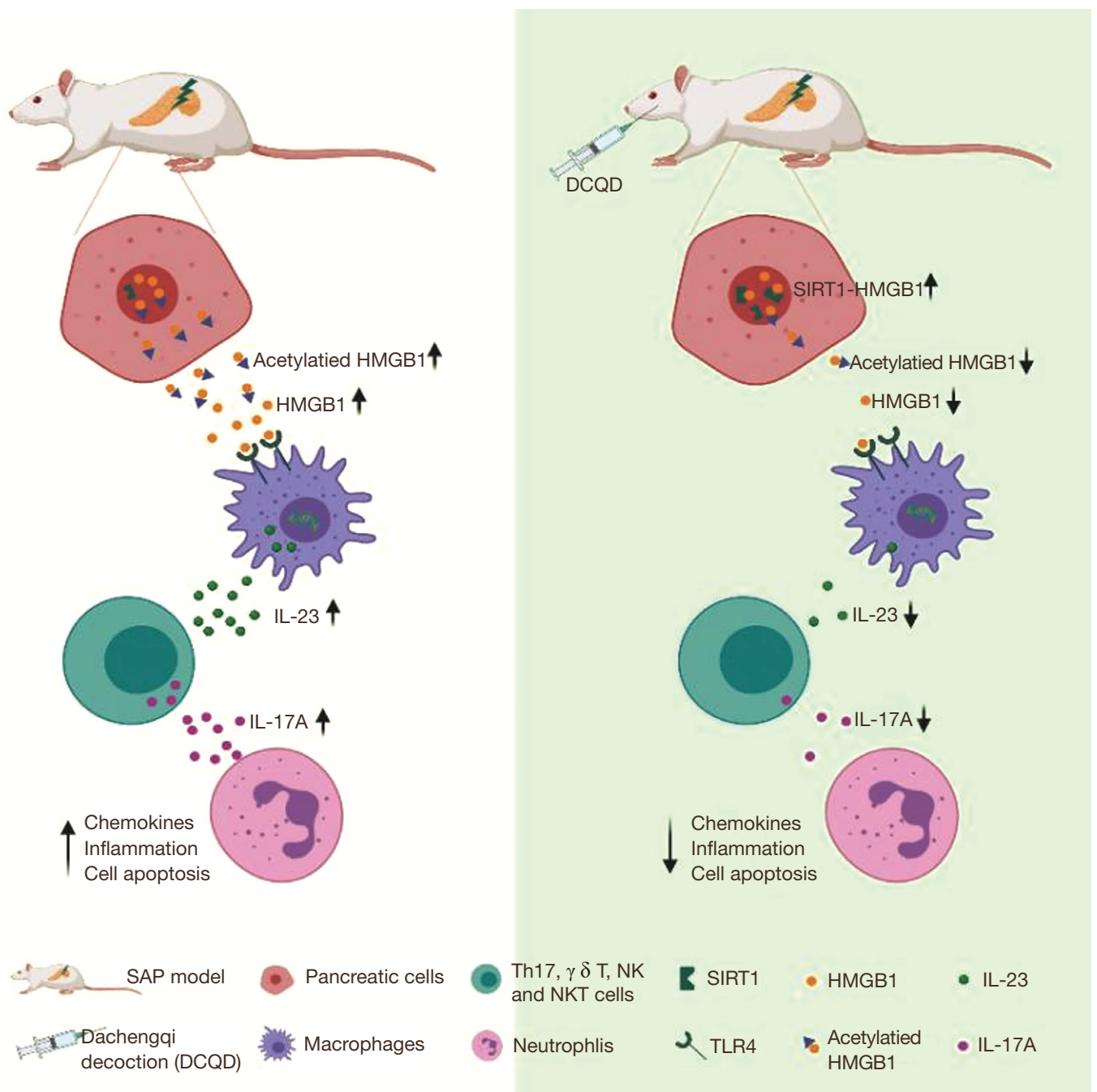

Figure 8 The signal pathway of DCQD on pancreatic microcirculatory in SAP. DCQD, Dachengqi decoction; SAP, severe acute pancreatitis.

that implied that the inhibitory effect of DCQD in neutrophil inactivation is through the inactivation of the IL-23/IL-17A axis through HMGB1.

HMGB1 is a non-DNA binding protein in the nucleus of eukaryotic cells and is widely distributed in the pancreas, lung, liver, kidney, lymph, and other tissues (2). It is known to function as a DAMP, interacting with members of RAGE and TLR (2). In our study, SAP only influences TLR-4. This is inconsistent with previous research attributed to drug dosage, purity, technology, and individual differences in rats. TLR-4 is a pattern-recognition receptor (PRR), and it has been extensively validated that TLR-4 mediates HMGB1-induced pancreatic injury in SAP (2). Previous research showed that HMGB1 stimulates the expression of TLR-4 in the pancreas, which is positively related to promoting macrophages and dendritic cells to secrete IL23 in SAP (8). Blocking TLR-4 can trigger the inactivation of IL-23/IL-17A signaling (18). In our in vivo study, DCQD treatment markedly advanced the inactivation of the HMGB1/TLR-4 signal pathway, as shown by a lower expression of TLR-4 and CD68, and lower acetylation, migration, and release of HMGB1. Furthermore, r-HMGB1 can abolish the impact of DCQD on the signal pathway of HMGB1/TLR-4 in SAP, as confirmed higher expression of TLR-4 and CD68 and greater acetylation, migration, and release of HMGB1 in the r-HMGB1 group than in the DCQD group.

In vitro studies further confirmed that DCQD could 
decrease HMGB1 secretion, and HMGB1 can promote macrophages to secrete IL-23 and increase IL-23 and TLR-4 expression. In addition, SiRNA TLR-4 can advance macrophage inactivation and alleviate IL-23 and TLR4 expression. However, overexpression of TLR-4 has the opposite influence, as confirmed higher expression of LR-4 and IL-23 in the plasmid group than in the SAP group. The above results indicated that the negative effect of DCQD on the activation of IL-23/IL-17A signaling is through the promotion of the inactivation of the HMGB1-TLR-4 signal pathway through deacetylation of HMGB1.

Noteworthy, the degree of deacetylation of HMGB1 is dependent on SIRT1, one of NAD + dependent histone deacetylase, which can deacetylate the activities of HMGB1 through interacting with HMGB1 at promoter region (19). In the study, DCQD can suppress the sodium taurocholate or cerulean-induced decrease in SIRT1 activity, expression, and interaction of HMGB1. However, EX527 can eliminate the influence of DCQD on the SIRT-HMGB1 axis in SAP. This indicated that DCQD could alleviate HMGB1 acetylation by regulating the SIRT-HMGB1 signaling pathway in SAP.

In conclusion, our study provides compelling evidence that the protective effect of DCQD on the pancreatic microcirculatory system in SAP is through inhibiting neutrophil-mediated inflammatory reactions. The above action mechanism of DCQD is likely associated with the inactivation of the HMGB1-TLR-4-IL-23-IL-17A axis by targeting SIRT1. However, this study was only performed on non-knockout mice with small sample size. More studies are required with larger sample sizes, knockout mice and clinical observations, and more cellular intervention to confirm the efficacy and mechanism of DCQD in SAP.

\section{Acknowledgments}

Funding: The study was funded by the Foundation of Sichuan Provincial People's Hospital (No. 2020LY07, No. 2017LY11), and scientific research project of Sichuan Provincial Department of Science and Technology (No. 2020YJ0179).

\section{Footnote}

Reporting Checklist: The authors have completed the ARRIVE reporting checklist. Available at https://dx.doi. org/10.21037/gs-21-655
Data Sharing Statement: Available at https://dx.doi. org/10.21037/gs-21-655

Conflicts of Interest: All authors have completed the ICMJE uniform disclosure form (available at https://dx.doi. org/10.21037/gs-21-655). Dr. JW reported that the study was funded by the Foundation of Sichuan Provincial People's Hospital (No. 2017LY11). Dr. JZ reported that the study was funded by the Foundation of Sichuan Provincial People's Hospital (No. 2020LY07) and scientific research project of Sichuan Provincial Department of Science and Technology (No. 2020YJ0179). The other authors have no conflicts of interest to declare.

Etbical Statement: The authors are accountable for all aspects of the work in ensuring that questions related to the accuracy or integrity of any part of the work are appropriately investigated and resolved. All animal procedures were approved by the animal care committee of the Sichuan Provincial People's Hospital (No. 2011220), and the experimental protocols were strictly carried out based on NIH Guidelines for the care and use of animals.

Open Access Statement: This is an Open Access article distributed in accordance with the Creative Commons Attribution-NonCommercial-NoDerivs 4.0 International License (CC BY-NC-ND 4.0), which permits the noncommercial replication and distribution of the article with the strict proviso that no changes or edits are made and the original work is properly cited (including links to both the formal publication through the relevant DOI and the license). See: https://creativecommons.org/licenses/by-nc-nd/4.0/.

\section{References}

1. Wu BU, Johannes RS, Sun X, et al. The early prediction of mortality in acute pancreatitis: a large population-based study. Gut 2008;57:1698-703.

2. Broggi A, Granucci F. Microbe- and danger-induced inflammation. Mol Immunol 2015;63:127-33.

3. Leppkes M, Maueröder C, Hirth S, et al. Externalized decondensed neutrophil chromatin occludes pancreatic ducts and drives pancreatitis. Nat Commun 2016;7:10973.

4. Shen $X$, Li WQ. High-mobility group box 1 protein and its role in severe acute pancreatitis. World J Gastroenterol 2015;21:1424-35.

5. Yan B, Chen F, Xu L, et al. HMGB1-TLR4-IL23- 
IL17A axis promotes paraquat-induced acute lung injury by mediating neutrophil infiltration in mice. Sci Rep 2017;7:597.

6. Ni J, Hu G, Xiong J, et al. Involvement of interleukin-17A in pancreatic damage in rat experimental acute necrotizing pancreatitis. Inflammation 2013;36:53-65.

7. Zhang MJ, Zhang GL, Yuan WB, et al. Treatment of abdominal compartment syndrome in severe acute pancreatitis patients with traditional Chinese medicine. World J Gastroenterol 2008;14:3574-8.

8. Chen Z, Chen Y, Pan L, et al. Dachengqi Decoction Attenuates Inflammatory Response via Inhibiting HMGB1 Mediated NF- B and P38 MAPK Signaling Pathways in Severe Acute Pancreatitis. Cell Physiol Biochem 2015;37:1379-89.

9. Wang J, Chen G, Gong H, et al. Amelioration of experimental acute pancreatitis with Dachengqi Decoction via regulation of necrosis-apoptosis switch in the pancreatic acinar cell. PLoS One 2012;7:e40160.

10. Huang J, Tian R, Yang Y, et al. The SIRT1 inhibitor EX527 suppresses mTOR activation and alleviates acute lung injury in mice with endotoxiemia. Innate Immun 2017;23:678-86.

11. Li G, Wu X, Yang L, et al. TLR4-mediated NF- B signaling pathway mediates HMGB1-induced pancreatic injury in mice with severe acute pancreatitis. Int $\mathrm{J}$ Mol Med 2016;37:99-107.

12. Hu X, Ma R, Lu J, et al. IL-23 Promotes Myocardial I/

Cite this article as: Wang J, Zou Y, Chang D, Hong DQ, Zhang J. Protective effect of Dachengqi decoction on the pancreatic microcirculatory system in severe acute pancreatitis by down-regulating HMGB-TLR-4-IL-23-IL-17A mediated neutrophil activation by targeting SIRT1. Gland Surg 2021;10(10):3030-3044. doi: 10.21037/gs-21-655
R Injury by Increasing the Inflammatory Responses and Oxidative Stress Reactions. Cell Physiol Biochem 2016;38:2163-72.

13. Sun J, Zhang S, Zhang X, et al. IL-17A is implicated in lipopolysaccharide-induced neuroinflammation and cognitive impairment in aged rats via microglial activation. J Neuroinflammation 2015;12:165.

14. Kolaczkowska E, Kubes P. Neutrophil recruitment and function in health and inflammation. Nat Rev Immunol 2013;13:159-75.

15. Xu P, Wang J, Yang ZW, et al. Regulatory roles of the $\mathrm{PI} 3 \mathrm{~K} /$ Akt signaling pathway in rats with severe acute pancreatitis. PLoS One 2013;8:e81767.

16. Cua DJ, Tato CM. Innate IL-17-producing cells: the sentinels of the immune system. Nat Rev Immunol 2010;10:479-89.

17. Zheng Y, Danilenko DM, Valdez P, et al. Interleukin-22, a $\mathrm{T}(\mathrm{H}) 17$ cytokine, mediates IL-23-induced dermal inflammation and acanthosis. Nature 2007;445:648-51.

18. Wang X, Sun R, Wei H, et al. High-mobility group box 1 (HMGB1)-Toll-like receptor (TLR)4-interleukin (IL)23-IL-17A axis in drug-induced damage-associated lethal hepatitis: Interaction of $\gamma \delta \mathrm{T}$ cells with macrophages. Hepatology 2013;57:373-84.

19. Wei S, Gao Y, Dai X, et al. SIRT1-mediated HMGB1 deacetylation suppresses sepsis-associated acute kidney injury. Am J Physiol Renal Physiol 2019;316:F20-31. 